\title{
A plasmaspheric cavity resonance in a longitudinally non-uniform plasmasphere
}

\author{
S. Fujita ${ }^{1}$ and M. Itonaga ${ }^{2}$ \\ ${ }^{1}$ Meteorological College, Kashiwa 277-0852 \\ ${ }^{2}$ Faculty of Education, Yamaguchi University, Yamaguchi 753-8531 \\ (Received December 20, 2002; Revised March 26, 2003; Accepted April 3, 2003)
}

\begin{abstract}
Power spectra of a plasmaspheric cavity resonance (strictly, a plasmaspheric virtual resonance) in a longitudinally non-uniform plasmasphere are calculated. It is shown that the spectra depend on longitude. Therefore, a cavity resonance mode can have local time depending spectra when the plasmasphere is non-uniform in a longitudinal direction. This fact concludes that the local time dependent peak frequencies of the mid- and low-latitude Pi2 pulsations discussed by Kosaka et al. (2002) are also explained by the cavity resonance model. We also discuss that the surface eigenmode can be a possible generation mechanism for Pi2 pulsations localized in a longitudinal direction.
\end{abstract}

Key words: Pi2 pulsation, non-uniform plasmasphere, cavity resonance.

\section{Introduction}

Pi2 pulsations, which are closely associated with a substorm onset, have been intensively studied by using ground magnetic observations (Yumoto et al., 2001 and references therein). Recent ground observations showed that the midand low-latitude Pi2 pulsations are different from the highlatitude ones. For example, the $210^{\circ}$ magnetic meridian chain observation revealed that the high-latitude $\mathrm{Pi} 2$ pulsations and the mid- and low-latitude Pi2 pulsations have longer and shorter periods, respectively (Li et al., 1998). As for the mid- and low-latitude Pi2 pulsations, they show essentially latitude-independent power spectra (Yeoman and Orr, 1989; Sutcliffe and Yumoto, 1989, 1991; Yumoto, 1990). These pulsations are interpreted as a global mode in the magnetosphere.

There are two ideas regarding to generation of the global mode in the magnetosphere. One is the cavity resonance model (Kivelson and Southwood, 1986) and another is the surface eigenmode model (Chen and Hasegawa, 1974). Let us explain the former model first. When there is a magnetospheric domain (a cavity) where the fast magnetosonic wave is confined, there appear eigen oscillations trapped in this domain. Since a single eigen oscillation has latitudinally independent frequency, this is a global mode. Kivelson and Southwood (1986) presented the idea of the magnetospheric cavity oscillation (the global cavity mode). The global cavity mode is the eigen oscillation trapped in the whole magnetosphere. Evidently, this global cavity mode is not consistent with the feature of the $\mathrm{Pi} 2$ pulsations stated above because the high-latitude Pi2 pulsations do not exhibit the global-mode feature. Next, Fujita and Glassmeier (1995) proposed another cavity mode that is trapped in the plasmasphere. Since the plasmasphere cavity does not perfectly confine energy of the fast magnetosonic wave within it, wave energy leaks from this cavity. Lee (1996) defined this type of the oscillation as the plasmaspheric virtual resonance (PVR). The PVR has been studied intensively since then (Itonaga $e t$ al., 1997a,b; Pekrides, 1997; Lee, 1998; Fujita et al., 2000,
2001, 2002). The second model, the surface eigenmode, was first studied by Chen and Hasegawa (1974). This surface eigenmode is formed transiently in a sharp boundary of plasmas. It is characteristic that this wave is evanescent (wave amplitude $\sim e^{-\kappa x}$ where $\kappa$ and $x$ mean the imaginary wave number and the coordinate perpendicular to the boundary). Unlike the cavity mode, the surface eigenmode exists in an open system.

Recently, Kosaka et al. (2002) revealed that the Pi2 pulsations have peak frequencies dependent on local time from observations of the mid- and low-latitude $\mathrm{Pi} 2$ pulsations. The peak frequency becomes higher in the dawnside and lower in the duskside. They insisted that those $\mathrm{Pi} 2$ pulsations are generated in the magnetosphere as the surface eigenmodes because they thought that the PVR has a power spectrum independent on longitude. However, there were no works supporting their conclusion. Only Lee et al. (2000) considered coupled oscillations in an elliptical plasmasphere stretched in the noon-midnight direction. Their focus was the field line resonance (FLR) mode, and they did not mention that the PVR has a longitudinally independent power spectrum when the plasmasphere is non-uniform in longitude. Therefore, we need to check this. In addition to this motivation, there is another reason why we need to investigate the PVR in a nonuniform plasmasphere. Namely, since the plasmasphere has a dusk bulge (Carpenter, 1970), the plasmasphere is actually non-uniform in longitudes. Consequently, it is necessary to present numerical results of the PVR in the longitudinally non-uniform plasmasphere.

\section{Numerical Analysis}

We simulate behavior of a MHD wave invoked by a sudden onset of a wave generator (a substitute for the current disruption of the substorm (Lui, 1996)) in a longitudinally non-uniform plasmasphere. We employ the numerical technique used in Fujita et al. (2001, 2002). Longitudinal width of the wave generator is $30^{\circ}$ and it is located at $L \sim 10 R_{e}$ in the midnight. The normalized ionospheric Pedersen current 


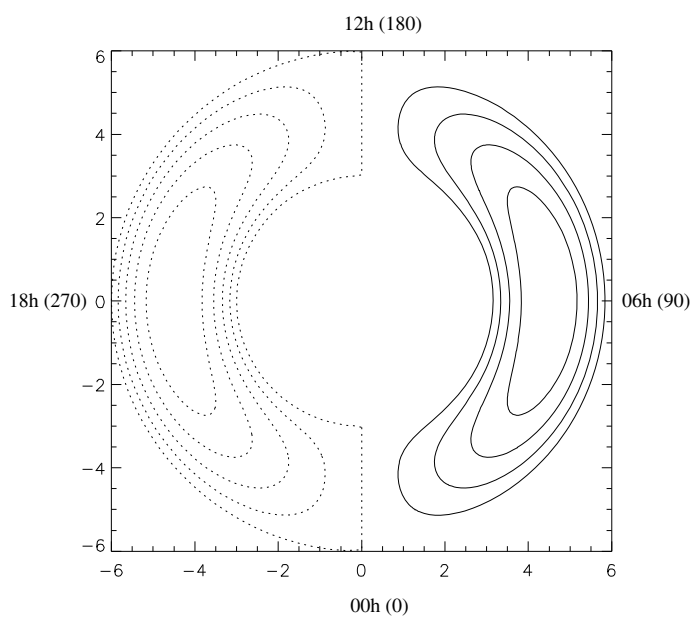

Fig. 1. Contours of the $V_{A}$-enhancement factor in the equatorial plane. This factor is given by Eq. (1). Full (broken) curves denote enhancement (depression). Contour interval is 0.025 . Numbers on the left axes and the bottom axes denote distance in $R_{e}$. Local time and corresponding $\varphi$ are also indicated. $V_{A}$ is enhanced in the dawnside (rightside in the figure) and depressed in the duskside (leftside).

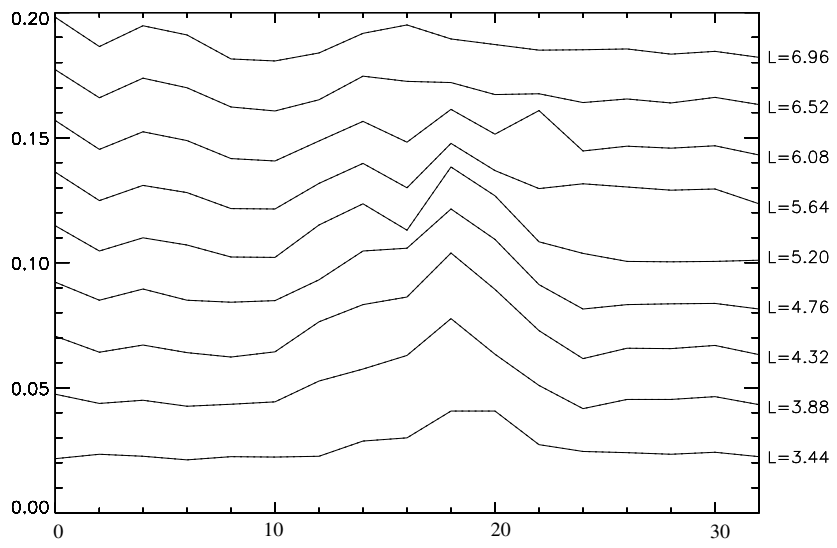

Fig. 2. Power spectra of $\delta E_{\varphi}$ in the equatorial plane at $18 \mathrm{~h}$ LT from $L$ $=3.44 R_{e}$ to $6.96 R_{e}$ in a longitudinally uniform plasmasphere. Power is shown in an arbitrary unit. Numbers in the horizontal axis are frequencies in $\mathrm{mHz}$.

is 1 . The model magnetosphere is essentially the same as that used by Fujita et al. (2002) except that the Alfvén speed $\left(V_{A}\right)$ in the plasmasphere ( $L \leq L_{p p}$ where $L_{p p}$ is the $L$ value at the plasmapause, $6 R_{e}$ ) exhibits longitudinal change as

$$
\begin{aligned}
& V_{A}(L, \varphi)=V_{A 0}(L) \\
& \times\left(1+\alpha \frac{\left(L-L_{i n}\right)\left(L_{p p}-L\right)}{L_{p p}^{2}} \sin \varphi\right),
\end{aligned}
$$

where $V_{A 0}(L)$ is the profile of $V_{A}$ used by Fujita et al. (2001, 2002). $\varphi$ is the local time in degrees and $L_{i n}$ is the $L$-value of the inner boundary $\left(3 R_{e}\right) . \alpha$ is an indicator expressing longitudinal non-uniformity. In the present calculation, we assign $\alpha$ as 2 . Therefore, the non-uniform factor in $V_{A}$ given by Eq. (1) ranges from $7 / 8$ at $\varphi=270^{\circ}$ (18h LT) to $9 / 8$ at $90^{\circ}(06 \mathrm{~h} \mathrm{LT})$ at $L=\left(L_{i n}+L_{p p}\right) / 2\left(=4.5 R_{e}\right)$. The longitudinal non-uniform factor is shown in Fig. 1. Note that

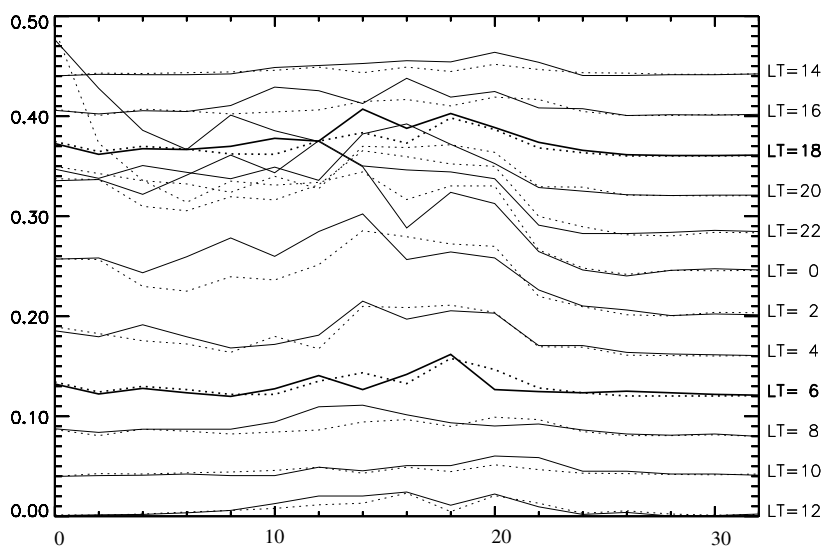

Fig. 3. Local time dependence of power spectra of $\delta E_{\varphi}$ in the equatorial plane at $L=5.20 R_{e}$. Full and broken curves denote the spectra for the non-uniform case and the uniform case, respectively. Power is shown in an arbitrary unit. Numbers in the horizontal axis are frequencies in $\mathrm{mHz}$.

this model is quite artificial. For example, the plasmasphere in the present model does not have a dusk bulge. This difference does not seem to bring about essential difference in frequency spectra of the global modes because the bulge is equivalently replaced by decrease in $V_{A}$ in the dusk side. The bulge widens radius of the plasmasphere, whereas the present $V_{A}$ distribution deepens plasmasphere depression of $V_{A}$. Both reduce effectively a local frequency of a radially trapped MHD wave (the frequency $\simeq V_{p s} / L_{p p}$ where $V_{p s}$ is an average of $V_{A}$ in the plasmasphere). In the present paper, we just intend to show influence of a longitudinal nonuniform plasmasphere to a MHD wave invoked by a localized wave generator. Therefore, we do not intend to assign a realistic plasmasphere.

First, before investigating power spectra of a simulated global mode in a longitudinally non-uniform plasmasphere, we show power spectra of the global mode in a uniform plasmasphere. Figure 2 presents the power spectra of $\delta E_{\varphi}$ (a longitudinal electric field component in a dipole coordinate system) at $18 \mathrm{~h} \mathrm{LT}$ in the region between $L=3.44 R_{e}$ and $6.96 R_{e}$. We use the FFT technique for $500 \mathrm{~s}$ data to calculate the power spectra. It is confirmed that the power spectra at $06 \mathrm{~h} \mathrm{LT}$ is identical to those at $18 \mathrm{~h} \mathrm{LT} \mathrm{(not} \mathrm{shown).}$ We notice from the figure that a spectral peak of about 18 $\mathrm{mHz}$ appears in $L=3.44 R_{e}$ to $6.08 R_{e}$ (the plasmasphere). The spectral peak is maximized near $L=5 R_{e}$ where $V_{A}$ is minimized. Therefore, this is a global mode with a constant frequency over latitudes. It is also noteworthy that the amplitude of this global mode remains significant near the inner boundary. This feature indicates that this wave is not a surface boundary mode confined near the plasmapause. As a result, it is possible to regard the global mode as a PVR. Therefore, we call this mode as a PVR from now.

Second, let us investigate local time dependence of the PVR frequency in a longitudinally non-uniform plasmasphere. The numerical results are shown in Fig. 3 which illustrates local time dependence of the power spectra of $\delta E_{\varphi}$ at $L=5.20 R_{e}$. The power spectra at $06 \mathrm{~h}$ LT (most enhanced $V_{A}$ ) and $18 \mathrm{~h} \mathrm{LT}$ (most depressed $V_{A}$ ) are drawn with thick 


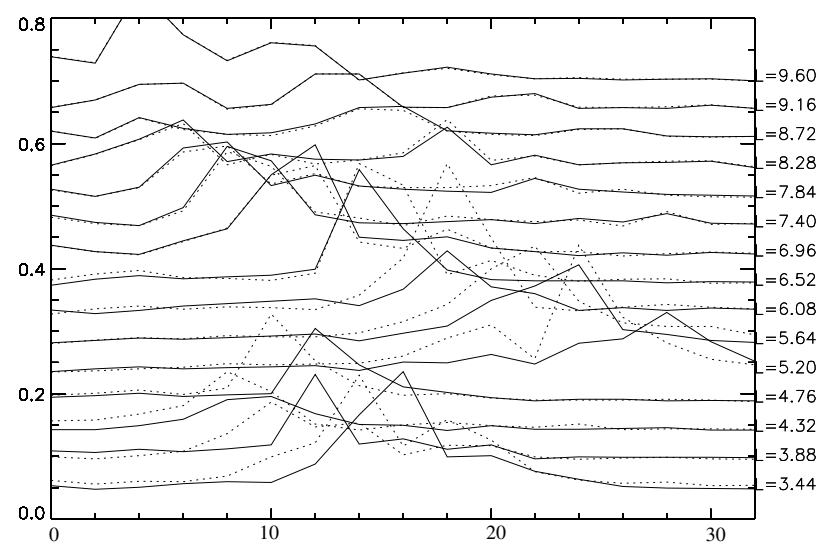

Fig. 4. Power spectra of $\delta E_{v}$ in the equatorial plane at 06h LT (full line) and at $18 \mathrm{~h}$ LT (broken line) from $L=3.44 R_{e}$ to $9.60 R_{e}$. Power is shown in an arbitrary unit. Numbers in the horizontal axis are frequencies in $\mathrm{mHz}$. The peak frequency is higher in the dawnside than in the duskside.

lines. The peak power appears at $18 \mathrm{mHz}$ at $06 \mathrm{~h} \mathrm{LT}$ and at $14 \mathrm{mHz}$ at $18 \mathrm{~h} \mathrm{LT}$. Therefore, it is evident that the frequency of the peak power is higher in the dawnside (06h LT) than in the duskside (18h LT). This result indicates that the PVR possesses the peak frequency dependent on the local time when the plasmasphere is non-uniform in a longitudinal direction. Therefore, local time variation of the $\mathrm{Pi} 2$ pulsations observed by Kosaka et al. (2002) can be interpreted within the cavity mode model.

Last, we investigate the localized mode (FLR). After Lee et al. (2000), the FLR is best reproduced in the component oriented relative to contours of constant $V_{A}$. Since equicontours of $V_{A}$ in our model are almost concentric circles, it is possible to show the FRL in $\delta E_{v}$ (a component directed to a curvature of an ambient dipole magnetic field). Thus, we illustrate in Fig. 4 power spectra of $\delta E_{v}$ at $06 \mathrm{~h} \mathrm{LT}$ and at $18 \mathrm{~h} \mathrm{LT}$ from $L=3.44 R_{e}$ to $9.60 R_{e}$. It exhibits peaks of the power at a specific frequency depending latitudes. This frequency is corresponding to the individual local field line resonance frequency. Compared the peak frequencies in the dawnside (06h LT) and in the duskside (18h LT), we notice that the former is higher than the latter in the plasmasphere $\left(L<6 R_{e}\right)$. This tendency is the same as that shown in $\delta E_{\varphi}$. This local time dependence of the peak frequency of the localized mode reflects $V_{A}$ distribution in the plasmasphere. Therefore, longitudinal variation of $V_{A}$ also affects behavior of the localized mode. This result is physically the same as that obtained by Lee et al. (2000).

Let us consider why the peak frequency of the PVR depends on longitude when the plasmasphere is non-uniform in longitude. For this purpose, we investigate eigenmodes in the plasmasphere, although the plasmasphere is not a perfect cavity. Namely, bearing in mind that behavior of the PVR is mainly controlled by structure of the plasmasphere, we can get information on the behavior of PVR from the plasmaspheric eigenmodes. In the plasmasphere with larger $V_{A}$ in the dawnside and smaller $V_{A}$ in the duskside, there are two kinds of the eigenmodes, one of which has larger intensity in the duskside and another has larger intensity in the dawnside. The former has a smaller frequency than the latter does.
Consequently, it is speculated that, at 18h LT, the plasmaspheric eigenmode with a smaller frequency of $14 \mathrm{mHz}$ is dominant. At 06h LT, the eigenmode with a larger frequency of $18 \mathrm{mHz}$ is dominant. This explanation is also valid for local time variation of the frequency of the localized mode. It is also expressed that the frequency of the eigenmode is proportional to $V_{A}$ in the region of the maximum amplitude divided by a scale size where the wave is effectively trapped.

\section{Discussion and Conclusions}

We present that the PVR in a longitudinally non-uniform plasmasphere shows a local time dependent peak frequency. The peak frequency tends to be lower in a region where $V_{A}$ is depressed. Depression of $V_{A}$ in the plasmasphere is equivalent to stretch of the plasmapause outward. Therefore, the region of smaller $V_{A}$ corresponds to the dusk bulge (Carpenter, 1970). Resultantly, the Pi2 pulsations tend to exhibit lower peak frequency in the duskside. This result is consistent with the observation by Kosaka et al. (2002). Note that the present plasmasphere model is too simplified one. Therefore, it is our next task to investigate the PVR in the realistic plasmasphere with the dusk bulge.

Then, we need to consider the surface eigenmode that was considered by Kosaka et al. (2002) as a possible generation mechanism of the mid- and low-latitude Pi2 pulsations with a local time dependent peak frequency. Both the cavity mode and the surface eigenmode belong to the fast magnetosonic wave that propagates isotropically with respect a direction of an ambient magnetic field. Essential difference between the two modes is derived from the condition where the wave is excited in a closed system or not. In other words, the cavity mode is a trapped wave that consists a foregoing wave and a reflected wave, whereas, the surface eigenmode consists of only the evanescent wave (the foregoing wave). Therefore, the surface eigenmode is expected to be detected when $\kappa^{-1}$ $\left(\kappa^{2}=-k_{x}^{2}\right.$ where $k_{x}$ is the wave number perpendicular to the plasmapause) is much smaller than an effective width $\left(r_{\text {cavity }}\right)$ of the plasmasphere cavity $\left(\kappa r_{\text {cavity }} \gg 1\right)$. In other words, the dispersion relation of the fast magnetosonic mode indicates that the wave with a smaller longitudinal extent (larger wave number along the surface of the plasmapause) tends to be the surface eigenmode.

Nosé et al. (2002) reported a Pi2 pulsations extended in the morning side of the plasmasphere. This observation indicates that the $\mathrm{Pi} 2$ pulsation has smaller wave number along the surface of the plasmapause. Therefore, it is not likely that the $\mathrm{Pi} 2$ pulsation is an evanescent wave in the outer plasmasphere. Namely, the Pi2 pulsation observed by Nosé et al. (2002) is regarded as the cavity mode, rather than the surface eigenmode. Unfortunately, observatories used in the analysis by Kosaka et al. (2002) are not so dense for confirmation of localized Pi2 pulsations. Although we do not confirm such a localized Pi2 pulsation, we should not omit possibility of the surface eigenmode because the $\mathrm{Pi} 2$ pulsation with a quite narrow longitudinal extent tends to be evanescent at the plasmapause. The $\mathrm{Pi} 2$ pulsations with a quite narrow longitudinal extent may exist, for example, if there is a waveguide from the source region to the plasmapause. Therefore, it is interesting to analyze multi-satellite data and the Circum-Pacific Magnetic Network (CPMN) data (Yu- 
moto et al., 2001) in order to investigate relation between the behavior of the mid- and low-latitude Pi2 pulsations and longitudinal extent of the pulsation.

As a summary, the main result of this paper is that the PVR has local time dependence of the frequency when the plasmasphere has a non-uniform structure in a longitudinal direction.

Acknowledgments. We are grateful to Iyemori and Nosé of Kyoto Univ. for valuable discussions. Numerical calculations were performed by the computer center of the National Institute of Polar Research and by the computer center of Nagoya University. This work was supported by the Grant-in-Aid for Scientific Research (c)(2) No. 14540415, from the Ministry of Education, Culture, Sports, Science and Technology.

\section{References}

Carpenter, D. L., Whistler evidence of the dynamic behavior of the duskside bulge in the plasmasphere. J. Geophys. Res., 75, 3837, 1970.

Chen, L. and A. Hasegawa, A theory of long-period magnetic pulsations, 2. Impulsive excitation of surface eigenmode, J. Geophys. Res., 79, 1033, 1974.

Fujita, S. and K.-H. Glassmeier, Magnetospheric cavity resonance oscillations with energy flow across the magnetopause, J. Geomag. Geoelectr., 47, 1277, 1995.

Fujita, S., M. Itonaga, and H. Nakata, Relation between the Pi2 pulsations and the localized impulsive current associated with the current disruption in the magnetosphere, Earth Planets Space, 52, 267, 2000.

Fujita, S., T. Mizuta, M. Itonaga, A. Yoshikawa, and H. Nakata, Transient MHD impulses in the magnetosphere-ionosphere system: The 2D model of the Pi2 pulsation, Geophys. Res. Lett., 28, 2161, 2001.

Fujita, S., M. Itonaga, A. Yoshikawa, H. Nakata, and T. Mizuta, A numerical simulation of the $\mathrm{Pi} 2$ pulsations associated with the substorm current wedge, J. Geophys. Res., 107(A3), 10.1029/2001JA000137, 2002.

Itonaga, M., A. Yoshikawa, and K. Yumoto, One-dimensional transient response of the inner magnetosphere at the magnetic equator, 1. Transfer function and poles, J. Geomag. Geoelectr., 49, 21, 1997a.

Itonaga, M., A. Yoshikawa, and K. Yumoto, One-dimensional transient re- sponse of the inner magnetosphere at the magnetic equator, 2. Analysis of waveform, J. Geomag. Geoelectr., 49, 49, 1997b.

Kivelson, M. G. and D. J. Southwood, Coupling of global magnetospheric MHD eigenmodes to field line resonances, J. Geophys. Res., 91, 4345, 1986.

Kosaka, K., T. Iyemori, M. Nosé, M. Bitterly, and J. Bitterly, Local time dependence of the dominant frequency of $\mathrm{Pi} 2$ pulsations at mid- and lowlatitudes, Earth Planets Space, 54, 771, 2002.

Lee, D.-H., Dynamics of MHD wave propagation in the low-latitude magnetosphere, J. Geophys. Res., 101, 15371, 1996.

Lee, D.-H., On the generation mechanism of Pi 2 pulsations in the magnetosphere, Geophys. Res. Lett., 25, 583, 1998.

Lee, D.-H., R. L. Lysak, and Y. Song, Field line resonances in a nonaxisymmetric magnetic field, J. Geophys. Res., 105, 10703, 2000

Li, Y., K. Yumoto, M. Itonaga, M. Shinohara, T.-I. Kitamura, and CPMN group, Equatorial Pi2's as indicators of substorms and the relation between dayside and nightside Pi2's, in SUBSTORMS-4, edited by S. Kokubun and Y. Kamide, pp. 555, 1998.

Lui, A. T. Y., Current disruption in the Earth's magnetosphere: Observations and models, J. Geophys. Res., 101, 13067, 1996.

Nosé, M., K. Takahashi, T. Uozumi, K. Yumoto, D. M. Milling, and P. R. Milling, Multi-point observations of a $\mathrm{Pi} 2$ pulsation on morning side, Proc. Sixth Int. Conf. on Substorms, edited by R. M. Winglee, 93, 2002.

Pekrides, H., A. D. M. Walker, and P. R. Sutcliff, Global modeling of Pi 2 pulsations, J. Geophys. Res., 102, 14343, 1997.

Sutcliffe, P. R. and K. Yumoto, Dayside Pi 2 pulsations at low latitudes, Geophys. Res. Lett., 16, 887, 1989.

Sutcliffe, P. R. and K. Yumoto, On the cavity mode nature of low-latitude Pi 2 pulsations, J. Geophys. Res., 96, 1543, 1991.

Yeoman, T. K. and D. Orr, Phase and spectral power of mid-latitude Pi 2 pulsations: Evidence for a plasmaspheric cavity resonance, Planet. Space Sci., 37, 1367, 1989.

Yumoto, K., Evidences of magnetospheric cavity Pi 2 waves, J. Geomag. Geoelectr., 42, 1281, 1990 .

Yumoto, K. and CPMN group, Characteristics of Pi2 geomagnetic pulsations observed at the CPMN stations: A review of the STEP results, Earth Planets Space, 53, 981, 2001.

S. Fujita (e-mail: sfujita@mc-jma.ac.jp) and M. Itonaga 